\title{
LUPUS-LIKE SYNDROME INDUCED BY UTERINE SARCOMA: A CASE REPORT
}

Andrea Worm Furtado 1,*, Afonso Guilherme Schmidt', Larissa Vargas Cruz', Ana Laura Fischer Kunzler', Victoria Silveira De Carvalho', Maurício Simoni Candaten', Vanessa Hax', Odirlei André Monticielo', Ricardo Machado Xavier'

1. Hospital de Clínicas de Porto Alegre, Porto Alegre (RS), Brazil.

*Corresponding author: andreawfurtado@yahoo.com.br

\section{BACKGROUND}

Uterine sarcomas are rare malignancies, corresponding to $3 \%$ of uterine tumors. Signs and symptoms typically include abnormal uterine bleeding, abdominal pain and bloating, and abdominal mass. In medical practice, the diagnosis of uterine sarcomas is suspected in postmenopausal women with presumed diagnosis of leiomyoma with indication for hysterectomy to symptomatic relief. We report a case of uterine sarcoma with unusual systemic lupus erythematosus (SLE)-like features.

\section{CASE REPORT}

A 54-year-old female patient, previously healthy, attended the rheumatology service, with a five-month history of daily fever, erythematous skin lesions, fatigue, dry cough and weight loss of $10 \mathrm{~kg}$. Upon physical examination, there was Raynaud's phenomenon and photosensitivity. Complementary exams showed anemia, increased acute phase reactants (APR), antinuclear factor 1/360 nuclear fine speckled and 1/160 nucleolar, presence of lupus anticoagulant, pericardial effusion and traces of protein in urine analysis. Abdominal computed tomography (CT) showed leiomyomatosis. No other abnormalities were detected on paranasal sinuses and chest CT, bronchoscopy, upper digestive endoscopy, blood and urine culture, or serology for other infectious agents. Following presumed diagnoses of SLE, the patient received prednisone $40 \mathrm{mg} /$ day for 30 days before hospitalization. On hospital admission, although fever had disappeared, new pelvic mass was identified, as well as increased APR, expressed by C-reactive protein (CRP) of $259 \mathrm{mg} / \mathrm{L}$ and erythrocyte sedimentation rate (ESR) of $104 \mathrm{~mm} / \mathrm{h}$. Abdominal CT also showed a large expansive uterine lesion with central necrosis. Hysterectomy was performed, which led to anatomopathological diagnosis of undifferentiated uterine sarcoma. After surgery, the patient presented complete resolution of the clinical and laboratory findings, including $\mathrm{CRP}<6 \mathrm{mg} / \mathrm{L}$ and ESR of $24 \mathrm{~mm} / \mathrm{h}$.

\section{CONCLUSION}

The diagnosis of SLE depends on the form of presentation and the exclusion of alternative diagnoses. The 2019 ACR/EULAR classification criteria may help clinicians to systematically document each clinical domain of disease features, but it also demonstrates limited sensitivity and specificity to diagnostic purposes. Although malignancy is an important differential diagnosis, eventually the suspected primary lesion may not be clinically evident at first. There are few case reports in medical literature that describe uterine sarcoma manifesting fever of undetermined origin. However, this is the first study to describe unusual manifestations of uterine sarcoma similar to SLE. 